et al had occult adenocarcinomas of the large bowel. ${ }^{3}$ An association with neoplasm of the small bowel has not been described. In this case portasystemic shunting and diabetes mellitus may have been additional predisposing factors.

Adenocarcinoma of the ileum is rare in this age group. ${ }^{+}$Its coexistence with cystic fibrosis may have been a chance happening, but we speculate that a combination of slowed small bowel transit, raised faecal bile acid concentrations, altered bowel flora, and low plasma selenium and vitamin E concentrations, all previously reported in cystic fibrosis, may predispose to the development of gastrointestinal malignancy. Any such predisposition would become more obvious as life expectancy in cystic fibrosis improves.

We thank Dr Philip De Costa of the histopathology department and Mr Graham Hutchinson of the microbiology department for their advice, and Miss S Hockley for her secretarial help. ANR is supported by the Frances and Augustus Newman Research Foundation.

1 Abdul-Karim FW, King TA, Dahms BB, Gauderer MW'L, Boat TF. Carcinoma A $1982 ; 82: 758-62$.

2 Davis TME, Sawicka EH. Adenocarcinoma in cystic fibrosis. Thorax 1985;40 $199-200$

3 Jendrzejewski JW, Jones SR, Newcombe RL, Gilbert DN. Nontraumatic clostridial myonecrosis. Am J Med 1978;65:542-6.

Office of Population Censuses and Surveys. Cancer statistics registrations 1980 OPCS Monitor 1983. (MB1 No 12)

(Accepted 20 March 1985)

Department of Cystic Fibrosis, Brompton Hospital, London SW3 6HP

ANDREW N REDINGTON, MB, MRCP, senior house officer

ROBERT SPRING, BA, MB, senior house officer

JOHN C BATTEN, MD, FRCP, consultant physician

Correspondence to: Dr A N Redington, Cardiothoracic Institute, Brompton Hospital, London SW 3 6HP.

\section{Intestinal perforation associated with Yersinia enterocolitica infection}

Yersinia enterocolitica was first recognised as a pathogen in man in 1939.' It is a Gram negative coccobacillus of worldwide distribution and produces a wide range of clinical manifestations. ${ }^{2} 3$ The commonest is a self limiting gastroenteritis, but more serious variants may occur. We describe two patients who required emergency operations for acute abdominal conditions associated with this infection.

\section{Case reports}

Case 1-A 7 year old Japanese boy presented with a two day history of abdominal pain, fever, and diarrhoea. On examination he had a fever $(38 \mathrm{C})$ and was dehydrated, and there was tenderness in the left iliac fossa. Gastroenteritis was diagnosed provisionally, and treatment comprised intravenous rehydration. The symptoms continued for seven days, and when blood cultures yielded Bacteroides fragilis intravenous gentamicin and metronidazole were started. There was little clinical response, and a laparotomy four days later showed a pelvic abscess secondary to a perforated appendix After appendicectomy and drainage he made an uneventful recovery. Subsequently an antibody titre of $1 / 320$ against $Y$ enterocolitica type $\mathrm{O} 3$ was detected in blood obtained on the 10th day of the illness.

Case 2-A 14 year old English girl presented in a critically ill condition. She had been unwell for three weeks with diarrhoea, vomiting, abdomina pain, fever, and weight loss $(9.5 \mathrm{~kg})$. Two days before her admission the diarrhoea had suddenly stopped. On examination she had a fever (38 C), was dehydrated, malnourished (plasma albumin concentration $17 \mathrm{~g} / \mathrm{l}$ ), hypotensive and had a tachycardia. The abdomen was massively distended, and an $x$ ray film showed intestinal obstruction. After resuscitation laparotomy showed mechanical obstruction of the small bowel due to an inflammatory mass in the pelvis associated with numerous perforating ulcers in the ileum. A similar mass in the upper abdomen was related to jejunal ulceration. Two resections with primary anastomoses were performed to relieve the obstruction and restore intestinal continuity. Postoperatively, despite tota parenteral nutrition and intravenous penicillin, gentamicin, and metronidazole she was critically ill for eight days with persistent fever and ileus. An antibody titre of $1 / 640$ against $Y$ enterocolitica type O3 was detected and chloramphenicol substituted for the previous antibiotics. She remained seriously ill for a further 11 days and improved only after the dosage was increased. Eventually she recovered completely and was discharged home
32 days after admission. Throughout the illness all blood cultures were sterile, rectal swabs grew no pathogens, and computed tomography of the abdomen postoperatively showed no localised collections. She received total parenteral nutrition for 26 days and chloramphenicol for 18 days (seven days at high dosage).

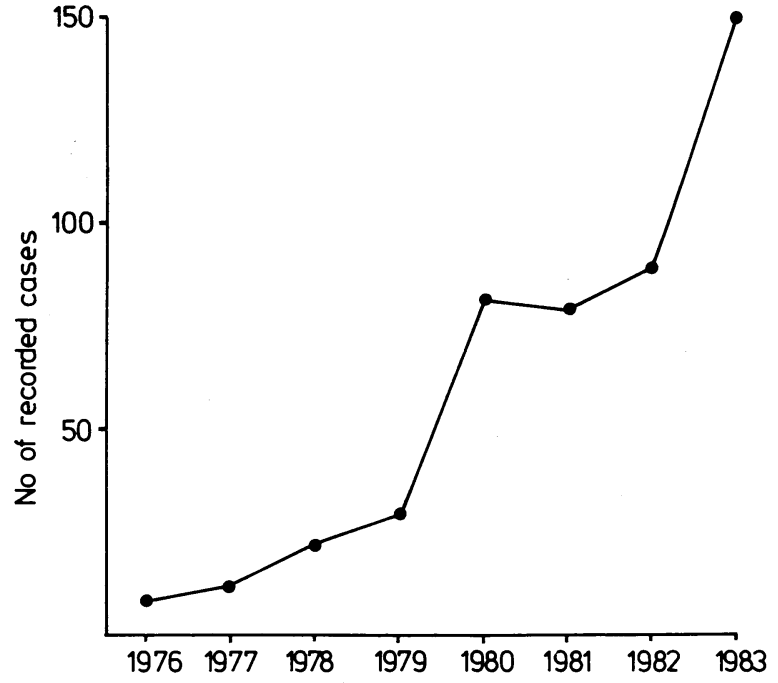

Incidence of positive diagnoses of Yersinia enterocolitica infection over past decade in United Kingdom. Diagnosis was made by serological examination or isolation from blood, stools, or lymph nodes, or both. (Courtesy of Dr C J Mitchell, Public Health Laboratory Service, Leicester.

\section{Comment}

The antibody titres against $Y$ enterocolitica, although measured in only single blood samples, were considered to be diagnostic by the Public Health Laboratory Service in Leicester (the British reference centre for this organism). During 1976-83 only 469 such infections were recorded by the Public Health Laboratory Service, and thus such infection appears to be either rare or underdiagnosed. The mean number of cases recorded each year increased from 18 (SD 9.5) in $1976-9$ to 100 (34) in 1980-3 (figure), but whether this reflects an increased incidence or greater awareness of the infection is uncertain. Yersinia is more prevalent abroad, ${ }^{2} 3$ but our patients must have contracted the infection within the United Kingdom as neither had been abroad recently.

The risks of yersinia infection are considerable; deaths have been recorded, ${ }^{4}$ and life threatening complications include acute appendicitis, ${ }^{2}$ septicaemia, ${ }^{2}{ }^{3}$ peritonitis, ${ }^{4}$ and gangrene of the small bowel. ${ }^{3}$ We suggest that yersinia may be a more important cause of acute abdominal conditions than is currently appreciated, and if antibody titres were obtained whenever acute intestinal problems were being investigated many more positive diagnoses would probably be made.

We thank Mr M J Notaras, Dr R T D Emond, and Professor K E F Hobbs for permission to report on their patients; Dr D Shove and Dr S Lucas for reporting on the pathology; and $\mathrm{Dr} \mathrm{C} J$ Mitchell and Mr E Fox, Public Health Laboratory Service, Leicester, for providing and permitting publication of the laboratory service data.

1 Schleifstein JI, Coleman MB. An unidentified microorganism resembling B lignieri and Past pseudotuberculosis, and pathogenic for man. NY State $\mathcal{F} \mathrm{Med}$ $1939 ; 39: 1749-53$.

2 Swaminathan MC, Harmon MC, Mehlman IJ. A review: Yersinia enterocolitica. f Appl Bacteriol 1982;52:151-83. 3 Anonymous. Yersiniosis today [Editorial]. Lancet $1984 ; \mathrm{i}: 84-5$.

with Yersinia enterocolitica. Arch Pathol 1974;98:17-22. with Yersinia enterocolitica. Arch Pathol $1974 ; 98: 17-22$.
eino RE, Renvall SY, Lipasti JA, Toivanen AM. Small bowel gangrene caused
by Yersinia enterocolitica III. Br Med $71980 ; 280: 1419$.

(Accepted 21 March 1985)

Barnet General Hospital, Barnet, Hertfordshire EN5 3DJ

GAVIN G P BROWNING, FRCSED, senior registrar in general surgery

Department of Infectious Diseases, Royal Free Hospital, London N10 1JN

WILLIAM R C WEIR, MRCP, senior registrar

Correspondence to: Dr W R C Weir, Hospital for Tropical Diseases, London NW 1. 